\title{
The abdominal wall hernia in cirrhotic patients: a historical challenge
}

\author{
Giuseppe Salamone ${ }^{* \dagger}$, Leo Licari ${ }^{\dagger}$, Giovanni Guercio, Sofia Campanella, Nicolò Falco, Gregorio Scerrino, \\ Sebastiano Bonventre, Girolamo Geraci, Gianfranco Cocorullo and Gaspare Gulotta
}

\begin{abstract}
Background: The incidence rate of abdominal wall hernia is $20-40 \%$ in cirrhotic patients. A surgical approach was originally performed only if complication signs and symptoms occurred. Several recent studies have demonstrated the usefulness of elective surgery. During recent decades, the indications for surgical timing have changed.

Methods: Cirrhotic patients with abdominal hernia who underwent surgical operation for abdominal wall hernia repair at the Policlinico "Paolo Giaccone" at Palermo University Hospital between January 2010 and September 2016 were identified in a prospective database, and the data collected were retrospectively reviewed; patients' medical and surgical records were collected from charts and surgical and intensive care unit (ICU) registries. Postoperative morbidity was determined through the Clavien-Dindo classification. Cirrhosis severity was estimated by the Child-PughTurcotte (CPT) score and MELD (model of end-stage liver disease) score. Postoperative mortality was considered up to 30 days after surgery. A follow-up period of at least 1 year was used to evaluate hernia recurrence.

Results: The univariate and multivariate analyses demonstrated the unique independent risk factors for the development of postsurgical morbidity (emergency surgery (OR 6.42; $p$ 0.023), CPT class C (OR 3.72; $p$ 0.041), American Society of Anesthesiologists (ASA) score $\geq 3$ (OR 4.72; $p$ 0.012) and MELD $\geq 20$ (OR 5.64; $p$ 0.009)) and postsurgical mortality (emergency surgery (OR 10.32; $p$ 0.021), CPT class C (OR 5.52; $p$ 0.014), ASA score $\geq 3$ (OR 8.65; $p$ 0.018), MELD $\geq 20$ (OR 2.15; $p$ 0.02)).

Conclusions: Concerning abdominal wall hernia repair in cirrhotic patients, the worst outcome is associated with emergency surgery and with uncontrolled disease. The correct timing of the surgical operation is elective surgery after ascites drainage and albumin/electrolyte serum level and coagulation alteration correction.
\end{abstract}

Keywords: Abdominal wall hernia, Cirrhosis, Surgery, Emergency, Risk factors

\section{Background}

The overall incidence of abdominal wall hernias is approximately $14 \%$; it increases to $20 \%$ in cirrhotic patients and might be up to $40 \%$ in cases of major ascites $[1,2]$. Factors such as weakness of the fascia and of the abdominal muscles due to malnutrition state and enlargement of pre-existing openings in the fascia promoted by increased abdominal pressure as a result of ascites formation are important contributors to the development of the hernias $[3,4]$. The watch-and-wait policy was commonly accepted in the past because of the high perioperative morbidity and

\footnotetext{
* Correspondence: lele.licari@gmail.com

'Salamone Giuseppe and Licari Leo contributed equally to this work.

Department of Surgical, Oncological and Oral Science, University of Palermo,

Policlinico P. Giaccone. Via Liborio Giuffré 5, 90127 Palermo, Italy
}

mortality that cirrhotic patients encountered. A surgical approach was then performed only if complication signs and symptoms occurred. However, the recommendations have changed during the last decade [5-7]. Previous retrospective studies [8] demonstrated that conservative treatment of abdominal wall hernias in cirrhotic patients is associated with considerable morbidity and mortality. Optimizing the patients with liver cirrhosis before elective hernia repair is critical for minimizing postoperative complications and reducing recurrence.

Moreover, it is commonly accepted that abdominal wall hernia repair should ideally be performed during liver transplantation or during liver function improvement.

In candidates for liver transplantation, the surgical operation should be performed during transplantation 
unless the patient presents with significant symptoms or hernia complications or if the perspective to be transplanted exceeds 3-6 months [9].

Several studies have demonstrated that elective surgery in cirrhotic patients could be safe, even when refractory ascites or advanced cirrhosis is diagnosed, if it is performed in a high-volume liver center [9-11]. Early elective hernia repair in these patients should be advocated considering the hepatic reserve and the patient's condition. The study herein analyzed the characteristics of cirrhotic patients who underwent abdominal wall hernia repair and investigated the risk factors for postoperative morbidity and mortality.

\section{Methods}

Cirrhotic patients with abdominal hernia who underwent surgical operation for abdominal wall hernia repair at the Policlinico "Paolo Giaccone" at Palermo University Hospital between January 2010 and September 2016 were identified in a prospective database, and the data collected were retrospectively reviewed; patients' medical and surgical records were collected from charts and the surgical and ICU registries.

The diagnosis of abdominal wall hernia was obtained after physical examination and US/CT scan execution.

Cirrhosis was documented by anamnestic data and confirmed through clinical, laboratory, and radiological findings.

Postoperative morbidity was determined through the Clavien-Dindo classification; classes III to V events were considered major complications. Cirrhosis severity was estimated by the Child-Pugh-Turcotte (CPT) score and MELD score calculated at the time of the surgical procedure.

Postoperative mortality was considered up to 30 days after surgery [12-14]. A follow-up period of at least 1 year was used to evaluate hernia recurrence, as diagnosed with physical examination and US/CT scan.

Patients with refractory ascites underwent paracentesis, albumin and serum electrolytes were replaced, nutritional support was guaranteed, and coagulation disorders were corrected pre- and postoperatively when indicated.

Abdominal wall hernias were repaired with the direct suture repair surgical technique when the defect did not exceed $3 \mathrm{~cm}$ or in cases of a contaminated/dirty surgical field; otherwise, the mesh-repair technique was adopted with sublay retromuscular positioning of a polyester mesh fixed at the posterior fascia of the rectus abdominis muscle with non-reabsorbable sutures. Indirect inguinal hernias were repaired with the plug- and mesh-mediated technique using a polypropylene plug fixed in the internal inguinal ring at the conjoint tendon and Cooper ligament; the polypropylene mesh was then positioned under the aponeurosis of the external oblique muscle (Trabucco technique). Direct inguinal hernias were repaired by performing the Lichtenstein technique after sac isolation and inversion into the preperitoneal space, preserving the integrity of the peritoneal sac. Patients with a diagnosis of recurrent abdominal wall hernia were not enrolled in the database. All surgical operations were performed via laparotomy.

Patient characteristics are shown in Table 1.

Twenty-six patients (22\%) were operated on under general anesthesia; 83 patients (71\%) underwent the surgical operation under local anesthesia, and eight patients (7\%) had spinal anesthesia. Details regarding the distribution of the type of hernia, anesthesia regimen used for each surgical operation and mesh usage are shown in Table 1.

\section{Statistical analyses}

Data were analyzed using Excel 2013 and IBM SPSS software, version 21 . The median was obtained for continuous variables. Comparisons of continuous variables were made using Student's $t$ test or the Mann-Whitney test, where appropriate. Comparisons of categorical variables were made with the chi-squared $\left(x^{2}\right)$ test or Fisher's exact test. The statistical significance level was set to $p$ value $<0.05$.

Univariate analysis for morbidity and survival was performed; the clinical variables included were emergency, CPT, ASA score, ascites, prosthesis use, MELD score, age, sex, and general anesthesia; the type of hernias was evaluated in the univariate analysis to identify possible risk factors for postoperative morbidity. The variables with $p$ values $<0.05$ in the univariate analysis were included in the multivariate logistic regression, considering odds ratios with $95 \%$ confidence intervals and $p$ values $<0.05$.

\section{Results}

Between January 2010 and September 2016, 117 cirrhotic patients were identified as undergoing abdominal wall hernia repair. Forty-one patients $(35 \%$ of the cirrhotic patients with abdominal wall hernia) were treated in emergency situations. The median pre-operative MELD score was 13 . The MELD score rate $\geq 20$ in elective and emergency surgery was $20 \%$ and $41 \%$ respectively (Table 1). The minimal follow-up time was 1 year. Mesh positioning was performed in 76 cases, of which 21 were in the emergency group. Emergency criteria were perforation $(n=4)$, incarceration $(n=27)$, strangulation $(n=7)$, and skin ulceration $(n=3)$.

Six patients had bilateral inguinal hernia, 30 had monolateral inguinal hernia, 60 had umbilical hernia, and 21 had incisional hernia.

Death occurred in 27 patients within 30 days after surgery and in 22 after emergency surgical operations; the causes of death were MOF due to sepsis after infection of the ascites $(n=16)$ and major ascites for decompensated 
Table 1 Characteristics of the population

\begin{tabular}{|c|c|c|c|c|}
\hline \multicolumn{2}{|c|}{ Variables } & Population $(n=117)$ & Elective surgery $(n=76)$ & Emergency surgery $(n=41)$ \\
\hline \multicolumn{2}{|l|}{ Age } & $60(53-81)$ & $60(53-81)$ & $65(60-81)$ \\
\hline \multicolumn{2}{|l|}{ Male } & $100(86 \%)$ & $66(88 \%)$ & $34(82 \%)$ \\
\hline \multicolumn{2}{|c|}{ Mean BMI } & 25 & 27 & 24 \\
\hline \multicolumn{2}{|c|}{ Poorly controlled ascites ( $n$ of patients) } & 41 & 14 & 27 \\
\hline & A ( $n$ of patients) & $41(35 \%)$ & $41(54 \%)$ & 0 \\
\hline & $\mathrm{B}$ ( $n$ of patients) & $27(23 \%)$ & $23(30 \%)$ & $4(10 \%)$ \\
\hline & $C$ ( $n$ of patients) & $49(42 \%)$ & $12(16 \%)$ & $37(90 \%)$ \\
\hline \multicolumn{2}{|c|}{ Mean pre-operative MELD score } & 13 & 12 & 16 \\
\hline \multicolumn{2}{|c|}{$\mathrm{n}$ of patients with MELD score $\geq 20$} & $32(27 \%)$ & $15(20 \%)$ & $17(41 \%)$ \\
\hline \multicolumn{2}{|c|}{ Mesh use } & $76(65 \%)$ & $55(47 \%)$ & $21(18 \%)$ \\
\hline \multicolumn{2}{|c|}{ Mean in-hospital stay (days) } & 10 & 7 & 16 \\
\hline \multicolumn{2}{|c|}{ Post-operative ICU (n of patients) } & $23(20 \%)$ & $8(10 \%)$ & $15(36 \%)$ \\
\hline \multicolumn{2}{|c|}{ Mean ICU stay (days) } & 7 & 2 & 10 \\
\hline \multicolumn{2}{|c|}{ Death } & $27(23 \%)$ & $5(6.6 \%)$ & $22(53.6 \%)$ \\
\hline \multirow{5}{*}{\multicolumn{2}{|c|}{ Clavien-Dindo score | }} & 27 & 27 & 0 \\
\hline & & 22 & 22 & 0 \\
\hline & & 31 & 22 & 9 \\
\hline & & 10 & 0 & 10 \\
\hline & & 27 & 5 & 22 \\
\hline \multicolumn{2}{|c|}{ ASA score $<3$} & $29(25 \%)$ & $29(38 \%)$ & $0(0 \%)$ \\
\hline \multicolumn{2}{|c|}{ ASA score $\geq 3$} & $88(75 \%)$ & 47 (62\%) & $41(100 \%)$ \\
\hline \multicolumn{2}{|c|}{ Bilateral hernia } & 6 & 4 & 2 \\
\hline \multicolumn{2}{|c|}{ Mesh use } & 6 & 4 & 2 \\
\hline \multicolumn{2}{|c|}{ General anesthesia } & $1(20 \%)$ & 0 & 1 \\
\hline \multicolumn{2}{|c|}{ Spinal anesthesia } & 5 (80\%) & 4 & 1 \\
\hline \multicolumn{2}{|c|}{ Monolateral hernia } & 30 & 19 & 11 \\
\hline \multicolumn{2}{|c|}{ Mesh use } & 30 & 19 & 11 \\
\hline \multicolumn{2}{|c|}{ General anesthesia } & $1(3 \%)$ & 0 & 1 \\
\hline \multicolumn{2}{|c|}{ Local anesthesia } & $26(87 \%)$ & 16 & 10 \\
\hline \multicolumn{2}{|c|}{ Spinal anesthesia } & $3(10 \%)$ & 3 & 0 \\
\hline \multicolumn{2}{|c|}{ Umbilical hernia } & 60 & 37 & 23 \\
\hline \multicolumn{2}{|c|}{ Mesh use } & 19 & 16 & 3 \\
\hline \multicolumn{2}{|c|}{ General anesthesia } & $3(5 \%)$ & 0 & 3 \\
\hline LoC & cal anesthesia & $57(95 \%)$ & 37 & 20 \\
\hline Incisic & nal hernia & 21 & 16 & 5 \\
\hline Me & sh use & 21 & 16 & 5 \\
\hline Ger & neral anesthesia & $21(100 \%)$ & 16 & 5 \\
\hline
\end{tabular}

cirrhosis $(n=6)$ with ascites leakage. In the elective group, death occurred after postoperative heart attack $(n=1)$, major ascites for decompensated cirrhosis $(n=2)$, and acute kidney failure $(n=2)$.

Emergency patients also presented with a markedly higher number of perioperative class III-V complications according to the Clavien-Dindo classification.
The median in-hospital stay was 10 days. Longer median hospital (16 vs. 7 days) and intensive care unit (10 vs. 2 days) stays were observed in the emergency patient group. Two hernia recurrences (monolateral indirect inguinal hernia-P2L according to European Hernia Society (EHS) classification-treated with Trabucco mesh repair and umbilical hernia treated with direct suture 
repair) were identified in the emergency group during the follow-up period; the umbilical hernia was then electively treated with the mesh-mediated pre-peritoneal open technique; the recurrent inguinal hernia was then electively treated by identifying the sac and the defect. The defect was diagnosed in the canal's posterior wall, describing a direct recurrent inguinal hernia, perhaps from displacement of the mesh from the pubic tubercle surface, where it was first anchored; a plug was then positioned in the defect after hernia inversion inside the abdominal cavity, reconstructing the anatomy of the inguinal canal.

The univariate analysis conducted to identify if the type of hernia could represent a risk factor for postoperative morbidity showed a considerable $p$ value for bilateral inguinal hernia $(p<0.01)$ and incisional hernia $(p<0.01)$ (Table 2) not confirmed on the multivariable analysis. In the same way, we demonstrated that general anesthesia had a considerable $p$ value in the univariate analysis for morbidity $(p=0.009)$ and mortality $(p=0.008)$ not confirmed on the multivariable analysis (Table 2).

The variables with $p$ values $<0.05$ in the univariate analysis were included in the multivariate logistic regression. The multivariate analysis conducted showed that emergency surgery, CPT class C, ASA score $\geq 3$, and

Table 2 Univariate and multivariate logistic regression analyses for morbidity

\begin{tabular}{llll}
\hline & OR & $95 \% \mathrm{Cl}$ & $p$ \\
\hline Univariate & & & \\
Emergency & 11.62 & $3.23-40.76$ & 0.026 \\
CPT C & 10.49 & $2.16-32.43$ & 0.003 \\
ASA score $\geq 3$ & 9.43 & $2.01-25.57$ & 0.001 \\
Ascites & 0.76 & $0.23-50.78$ & 0.114 \\
Mesh use & 0.54 & $0.57-70.87$ & 0.214 \\
MELD score $\geq 20$ & 2.06 & $2.41-22.76$ & 0.017 \\
Age $>$ 60 & 1.53 & $0.12-56.19$ & 0.421 \\
Male & 1.54 & $0.09-12.93$ & 0.769 \\
Bilateral inguinal hernia & 7.62 & $5.32-30.11$ & 0.009 \\
Monolateral inguinal hernia & 3.23 & $0.82-11.41$ & 0.11 \\
Umbilical hernia & 0.76 & $0.98-45.71$ & 0.23 \\
Incisional hernia & 1.82 & $2.92-7.34$ & 0.009 \\
General anesthesia & 4.32 & $2.76-80.91$ & 0.009 \\
Multivariate & & & \\
Emergency & 6.42 & $1.76-40.53$ & 0.023 \\
CPT C & 3.72 & $1.23-37.28$ & 0.041 \\
ASA score $\geq 3$ & 4.72 & $3.41-45.81$ & 0.012 \\
MELD score $\geq 20$ & 5.64 & $1.71-23.67$ & 0.009 \\
Bilateral inguinal hernia & 4.12 & $0.42-46.71$ & 0.17 \\
Incisional hernia & 6.75 & $0.67-52.86$ & 0.26 \\
General anesthesia & $0.12-34.22$ & 0.12 \\
\hline
\end{tabular}

MELD $\geq 20$ were unique independent risk factors for the development of postsurgical morbidity (Table 2) and mortality (Table 3).

\section{Discussion}

Traditionally, hernia repair in the presence of advanced cirrhosis and ascites has resulted in high rates of morbidity and mortality, prompting many surgeons to avoid elective repair and to operate only when complications develop. In 1960, Baron reported a mortality of $31 \%$ in a case series of 16 patients who underwent umbilical hernia repair and who had cirrhosis. O'Hara et al. reported a morbidity rate of $22 \%$ and a mortality of $16 \%$ in emergency surgery; these data suggest that surgical repair should be performed in uncomplicated hernias. The risk of treating complicated hernia conservatively heavily outweighs the risk of surgical repair. Non-operative management of complicated hernias with antibiotics and dressing changes might result in mortality rates in the range of $60-88 \%$. Therefore, complicated umbilical hernias in cirrhotic patients should be repaired emergently [15].

The complications related to surgical operations of abdominal wall hernias are high in cirrhotic patients, as much of the impact of abdominal hernia presence is on QoL. In recent decades, the indications for surgical timing and management have changed. The watch-and-wait strategy has been abandoned in favor of elective surgery. However, the indications for surgical repair of abdominal wall hernias in cirrhotic patients remain a controversial challenge.

Table 3 Univariate and multivariate logistic regression analyses for mortality

\begin{tabular}{llll}
\hline & OR & $95 \% \mathrm{Cl}$ & $p$ \\
\hline Univariate & & & \\
Emergency & 21.76 & $4.26-31.53$ & 0.003 \\
CPT C & 3.56 & $13.21-76.32$ & 0.001 \\
ASA score $\geq 3$ & 10.31 & $3.54-16.32$ & 0.001 \\
Ascites & 2.45 & $0.22-67.21$ & 0.51 \\
Mesh use & 1.21 & $0.86-14.32$ & 0.41 \\
MELD score $\geq 20$ & 1.69 & $2.02-23.63$ & 0.017 \\
Age $>$ 60 & 2.47 & $0.63-45.61$ & 0.21 \\
Male & 0.12 & $0.03-10.45$ & 0.53 \\
General anesthesia & 2.43 & $5.28-43.61$ & 0.008 \\
Multivariate & & & \\
Emergency & 10.32 & $3.66-47.82$ & 0.021 \\
CPT C & 5.52 & $1.67-32.45$ & 0.014 \\
ASA score $\geq 3$ & 8.65 & $3.65-87.23$ & 0.018 \\
MELD score $\geq 20$ & 2.15 & $2.71-32.68$ & 0.002 \\
General anesthesia & 7.22 & $4.71-13.65$ & 0.23 \\
\hline
\end{tabular}


Expectant treatment of cirrhotic patients with abdominal wall hernia and ascites is associated with an increased rate of complications, such as incarceration, evisceration, ascites drainage, and peritonitis. These complications require emergency surgical treatment, which carries increased risk of morbidity and mortality. Conversely, elective hernia correction might be performed with fewer complications and is therefore advocated [9].

It has been demonstrated that the improved complication rates associated with modern surgical techniques and perioperative care justify the consideration of an early repair before complications occur. Kirkpatrick and Schubert reported improved outcomes and lower mortality in patients treated after 1975 than in patients treated prior to 1975 . A review by Maniatis and Hunt of papers published between 1956 and 1990 found a mortality rate of only $2 \%$ in the non-emergency setting, whereas the mortality rate was $14 \%$ with repair due to complications carried out as an emergency [16].

The data proposed suggest that emergency, CPT-C, ASA score $\geq 3$, and MELD score $\geq 20$ are risk factors for postoperative morbidity and mortality. In contrast, elective surgery appears to be successful and to be associated with lower mortality rate. Scientific reports indicate that adequate preparation of cirrhotic patients, with control of ascites, albumin and electrolytes serum levels, nutritional support, and coagulation patterns, allows for the success of elective surgery $[16,17]$.

The mortality rates for elective and emergency patients reported by our series were, respectively, 6.6 and $53.6 \%$. A reported mortality rate higher than that in the data published in the international literature can be explained not only by the high rate of MELD score $\geq 20$ in the two groups (20 and $41 \%$, respectively), but also by the high rate of CPT class $\mathrm{C}$ and ASA score $\geq 3$ that are respectively 16 and $62 \%$ in elective surgery group, 90 and $100 \%$ in emergency surgery group, as reported in Table 1.

Ascites control is essential to reducing perioperative complications and recurrence.

In the past, there was a considerable lack of evidence regarding how severe liver dysfunction must be to preclude operative repair. There did not appear to be any reliable, commonly accepted methods to determine whether the cirrhosis was too severe to allow for elective repair or was mild enough that the risk of major complications was low enough to justify the repair [16].

It has now been demonstrated that the CPT score and MELD score are the best ways to identify the severity of liver illness; these scores adequately correlate with prognostic evaluations of postoperative morbidity and mortality in cirrhotic patients.

Recently, although the MELD score was optimized for liver transplantation patients, it appears to be the most objective means to evaluate the surgical risk in cirrhotic patients. It has been demonstrated that a MELD score between 8 and 14 predicts poor surgical outcomes. Moreover, the worst outcome for abdominal surgery is described when the MELD score is above 20 [18-26]. The median pre-operative MELD score in our series was 13.

According to the multivariate analysis, elective surgery is preferable concerning the timing for hernia repair. Emergency surgery is strongly associated with a higher incidence of postoperative morbidity and mortality.

Refractory ascites is frequently associated with urgency even if it does not represent a risk factor in the multivariate analysis. Refractory ascites is surely considered a direct cause of a complicated hernia because of the increased abdominal pressure. This complication is also correlated with skin ulceration, risk of SSI, and ascites leaks.

\section{Conclusions}

The results of the data analysis show that performing the surgical operation of abdominal wall hernia repair in cirrhotic patients emergently is related to higher postoperative morbidity and mortality rates. This finding suggests that the correct timing of the surgical operation is elective surgery in controlled liver disease, monitoring the disease with the CPT score and the MELD score. An ASA score $\geq 3$ is also a risk factor for postoperative morbidity and mortality. All these risk factors should be considered in the prognostic evaluation of cirrhotic patients who require surgical operation for abdominal wall hernia repair. Furthermore, pre-operative refractory ascites should be managed with paracentesis and albumin/electrolytes serum level and coagulation alteration with appropriate correction.

\section{Acknowledgements}

We thank Dr. Comelli Albert, Department of Industrial and Digital Innovation, Policlinico P. Giaccone, University of Palermo, who performed the statistical analysis

The first and the second authors (Salamone G and Licari L) contributed to the writing of the manuscript in equal measure and should both therefore be considered first authors.

\section{Availability of data and materials}

The datasets used and/or analyzed during the current study are available from the corresponding author upon reasonable request.

\section{Authors' contributions}

$\mathrm{GS}, \mathrm{LL}$, and $\mathrm{GG}$ conceived of and designated the study. LL, SC, NF, GG, GS, $\mathrm{SB}$, and $\mathrm{GC}$ analyzed and interpreted the patient data. GS and LL were major contributors in writing the manuscript. GC and GG supervised the manuscript, discussed the results, and commented on the manuscript. GS and $\mathrm{LL}$ contributed to the design and implementation of the research, to the analysis of the results, and to the writing of the manuscript. All authors read and approved the final manuscript.

Ethics approval and consent to participate Not applicable 


\section{Consent for publication}

Not applicable

\section{Competing interests}

The authors declare that they have no competing interests.

\section{Publisher's Note}

Springer Nature remains neutral with regard to jurisdictional claims in published maps and institutional affiliations.

Received: 14 February 2018 Accepted: 18 July 2018

Published online: 28 July 2018

\section{References}

1. Belghiti J, Durand F. Abdominal wall hernias in the setting of cirrhosis. Semin Liver Dis. 1997;17:219-26.

2. Carbonell AM, Wolfe LG, DeMaria E. Poor outcomes in cirrhosis-associated hernia repair: a nationwide cohort study of 32,033 patients. Hernia. 2005;9:353-7.

3. Shlomovitz E, Quan D, Etemad-Rezai R, McAlister VC. Association of recanalization of the left ombilical vein with umbilical hernia in patients with liver disease. Liver Transpl. 2005;11:1298-9.

4. Garrison RN, Cryer HM, Howard DA, Polk HC. Classification of risk factors for abdominal operations in patients with hepatic cirrhosis. Ann Surg. 1984;199: 648-55.

5. Leonetti JP, Aranha GV, Wilkinson WA, et al. Umbilical herniorrhaphy in cirrhotic patients. Arch Surg. 1984;119:442-5.

6. O'Hara ET, Oliai A, Patek AJ, Nabseth DC. Management of umbilical hernias associated with hepatic cirrhosis and ascites. Ann Surg. 1975;181:85-7.

7. Arroyo A, García P, Pérez F, et al. Randomized clinical trial comparing suture and mesh repair of umbilical hernia in adults. Br J Surg. 2001;88:1321-3.

8. Gray SH, Vick CC, Graham LA, et al. Umbilical herniorrhapy in cirrhosis: improved outcomes with elective repair. J Gastrointest Surg. 2008;12:675-81.

9. Coelho JCU, Claus CMP, Campos ACL, et al. Umbilical hernia in patients with liver cirrhosis: a surgical challenge. World J Gastrointest Surg. 2016;8(7): 476-82

10. Andraus W, Sepulveda A, Pinheiro RS, et al. Management of uncommon hernias in cirrhotic patients. Transplant Proc. 2010;42:1724-8.

11. Park JK, Lee SH, Yoon WJ, et al. Evaluation of hernia repair operation in child-turcotte-pugh class c cirrhosis and refractory ascites. J Gastroenterol Hepatol. 2007;22:377-82

12. Pugh RN, Murray-Lyon IM, Dawson JL, et al. Transection of the oesophagus for bleeding oesophageal varices. Br J Surg. 1973;60:646-9.

13. Kamath PS, Wiesner $\mathrm{RH}$, Malinchoc $\mathrm{M}$, et al. A model to predict survival in patients with end-stage liver disease. Hepatology. 2001;33:464-70

14. Green SB. How many subjects does it take to do a regression analysis? Multivar Behav Res. 1991;26:499-510.

15. McKay A, Dixon E, Bathe O, Sutherland F. Umbilical hernia repair in the presence of cirrhosis and ascites: results of a survey and review of the literature. Hernia. 2009;13(5):461-8.

16. Belyansky I, Tsirline VB, Klima DA, et al. Prospective, comparative study of postoperative quality of life in tep, tapp, and modified lichtenstein repairs. Ann Surg. 2011;254:709-14. Discussion 714-705

17. Hansen JB, Thulstrup AM, Vilstup H, Sørensen HT. Danish nationwide cohort study of postoperative death in patients with liver cirrhosis undergoing hernia repair. Br J Surg. 2002;89:805-6

18. Hurst RD, Butler BN, Soybel DI, Wright HK. Management of groin hernias in patients with ascites. Ann Surg. 1992;216:696-700.

19. Eker $\mathrm{HH}$, van Ramshorst $\mathrm{GH}$, de Goede $\mathrm{B}$, et al. A prospective study on elective umbilical hernia repair in patients with liver cirrhosis and ascites. Surgery. 2011;150:542-6.

20. Hur YH, Kim JC, Kim DY, Kim SK, Park CY. Inguinal hernia repair in patients with liver cirrhosis accompanied by ascites. J Korean Surg Soc. 2011;80(6): 420-5.

21. Farnsworth N, Fagan SP, Berger DH, Awad SS. Child-Turcotte-Pugh versus MELD score as a predictor of outcome after elective and emergent surgery in cirrhotic patients. Am J Surg. 2004;188:580-3.

22. Befeler AS, Palmer DE, Hoffman M, et al. The safety of intra-abdominal surgery in patients with cirrhosis: model for end-stage liver disease score is superior to Child-Turcotte-Pugh classification in predicting outcome. Arch Surg. 2005;140:650-4. discussion 655
23. Teh SH, Nagorney DM, Stevens SR, et al. Risk factors for mortality after surgery in patients with cirrhosis. Gastroenterology. 2007:132:1261-9.

24. Arif $R$, Seppelt $P$, Schwill $S$, et al. Predictive risk factors for patients with cirrhosis undergoing heart surgery. Ann Thorac Surg. 2012;94:1947-52.

25. Neeff $H$, Mariaskin D, Spangenberg $H C$, et al. Perioperative mortality after non-hepatic general surgery in patients with liver cirrhosis: an analysis of 138 operations in the 2000s using child and meld scores. J Gastrointest Surg. 2011;15:1-11.

26. Maniatis AG, Hunt CM. Therapy for spontaneous umbilical hernia rupture. Am J Gastroenterol. 1995;90:310-2.

\section{Ready to submit your research? Choose BMC and benefit from:}

- fast, convenient online submission

- thorough peer review by experienced researchers in your field

- rapid publication on acceptance

- support for research data, including large and complex data types

- gold Open Access which fosters wider collaboration and increased citations

- maximum visibility for your research: over $100 \mathrm{M}$ website views per year

At BMC, research is always in progress.

Learn more biomedcentral.com/submissions 